\title{
Siphon flows and oscillations in long coronal loops due to Alfvén waves
}

\author{
R. Grappin ${ }^{1}$, J. Léorat ${ }^{1}$, and S. Rifai Habbal ${ }^{2,3}$ \\ ${ }^{1}$ Observatoire de Paris-Meudon, 1 place J. Janssen, 92195 Meudon Cedex, France \\ e-mail: [roland.grappin; jacques.leorat]@obspm.fr \\ 2 University of Wales, Aberystwyth SY23 3BZ, UK \\ ${ }^{3}$ Harvard-Smithsonian Center for Astrophysics, Cambridge, MA 02138, USA \\ e-mail: sdh@aber.ac.uk
}

Received 21 January 2005 / Accepted 16 March 2005

\begin{abstract}
Using an isothermal axisymmetric MHD model of the solar corona with transparent boundary conditions, we study how coronal loops with apex height between one third and two solar radii react to Alfvén waves generated at the base of the corona. The first response is a steady increase of the density along the excited loops. Density oscillations are also observed, along the apex of the longest loops, that is, those along which waves are substantially damped. Both the steady and the oscillating component scale as the square of the Alfvén wave amplitude, and accordingly, the frequency is double that of the mother wave. Siphon flows may also appear, along short or long loops, depending on whether both or a single foot-point of the loop system is excited.
\end{abstract}

Key words. Sun: corona - methods: numerical - magnetohydrodynamics (MHD)

\section{Introduction}

Observations obtained with SOHO and TRACE of the solar transition region and corona have shown that magnetic loops are characterized by temporal variability and significant plasma flows along the magnetic field lines. Evidence of catastrophic cooling and down-flows on the one hand, and of quasi-steady siphon flows on the other hand, have been reported (see review by Spadaro 2003). The siphon flows seem to be associated in particular with over-dense active region loops.

Stationary siphon flows have been analyzed theoretically for some time (Meyer \& Schmidt 1968). Robb \& Cally (1992) have analyzed how siphon flows (steady or unsteady) are controlled by pressure differences at the foot-points. Patsourakos et al. (2004) have shown the inability of steady flow models to explain the EUV coronal loops, while Spadaro et al. (2003) have been able to reproduce several features of the observed loops (in particular the over-densities) by applying transient heating at the chromospheric level.

In this paper, we consider Alfvén waves as a timedependent driver for pressure variations able to trigger siphon flows. Because we use a global coronal-solar wind model (see below), resolution constraints force us to concentrate on very long loops, with apex height between one third and two solar radii. Our motivation is two-fold. First, it has been noticed (but without detailed study of the mechanism) that siphon flows appeared spontaneously in equatorial loops, when Alfvén waves were directly injected in the corona in simulations of a global solar wind model (Grappin et al. 2002, hereafter GLH). Second, Alfvén waves are ubiquitous in the solar wind, and so it is expected that they propagate along closed loops also, not only in the open corona. Hence, their dynamical effect there should be studied.

Let us briefly recall their basic properties. Linearly polarized progressive (unidirectional) Alfvén waves exert a wave pressure, which is the part of the magnetic pressure associated with the fluctuating magnetic field. The average plasma acceleration is non zero, as soon as the wave amplitude decreases with distance along field lines, which may occur both due to stratification and/or to dissipation. This is the basic mechanism which allows Alfvén waves to push the solar wind along open magnetic field lines.

We are interested in this paper in the case of closed loops. Before discussing what happens in that case, we must specify the boundary conditions. If we believe that the dense photospheric layers determine completely (without feedback) the coronal motions, which is implicit in the often used "line-tied" hypothesis (see for instance Aulanier et al. 2004), then shaking the loop ends will accumulate energy in the loop system, lead to inflation of the whole coronal structure without producing the parallel flows we are interested in. If on the contrary we insist that the corona must exert a feedback, so that the loop ends are not strictly fixed to the photosphere, then the coronal boundary is at least partially transparent, and persistent parallel flows become possible. To really simulate this situation would require the inclusion of the transition between the corona and the 
photosphere within the numerical domain. In the present work, we consider the simpler framework of an isothermal corona and make the extreme assumption, opposite to the line-tied hypothesis, that the low coronal boundary is fully transparent: waves injected at one loop end will thus leave the loop without reflection when reaching the other end.

In this framework, is it possible to generate siphon flows in closed loops via Alfvén waves? Consider a symmetric loop with equal pressure at both foot-points (we do not rely on pre-existing pressure differences). Then, if one shakes one of the loop ends with Alfvén waves, the stratification will induce no pressure imbalance between foot-points, as it has symmetric effects on the wave amplitude in each loop branch. Dissipation is thus needed when shaking only one loop end. Actually, this is the only case that is easy to analyze. Consider now the case where both foot-points are excited with equal amplitudes (as in GLH). We need some additional ingredient to break the symmetry between the two foot-points in that case. Indeed, no siphon flows were observed in GLH, when the two waves injected are in phase, while a siphon flow was obtained when the phase is not equal. This is paradoxical, as the outcome should be, regardless of the phase difference, compressive waves with wave vectors parallel to the magnetic field lines, without the mean circulation which is called siphon flow. In particular, in the case of a one-dimensional model of a magnetic loop, these compressive waves rapidly leave the medium, leaving behind a stationary pattern of density balancing the magnetic pressure field associated with the pondero-motive force (Grappin et al. $2003 \mathrm{~b}$ ). How siphon flows emerge in the $2 \mathrm{D}$ case will be elucidated in the present paper, in which we consider in succession single foot-point excitation (to start with a not too complex situation), and then double foot-point excitation. We shall concentrate on the physical mechanism and will not attempt any quantitative fit of observational data.

We use the coronal-solar wind model of GLH (isothermal, axisymmetric MHD equations with transparent boundary conditions), in the heliocentric distance interval $[1,16] R_{\mathrm{s}}$, where the numerical resolution is concentrated in the coronal streamer region between one and four solar radii (Sect. 2). We then perform a systematic study of the siphon flow generation by Alfvén waves; we consider first exciting globally one or two hemispheres, and then groups of magnetic loops (Sect. 3). Section 4 contains the discussion.

\section{Numerics}

In the interior of the domain, we solve the MHD equations in spherical coordinates for density $\rho$, velocity $u_{r}, u_{\theta}, u_{\phi}$, azimuthal magnetic field $B_{\phi}$ and magnetic potential $\psi$ (the fields depending only on radial distance and latitude). The total magnetic field is:

$B=B^{0}+\operatorname{curl}\left(\psi e_{\phi}\right)+B_{\phi} e_{\phi}$

where $B^{0}$ is an external given dipole. On the other hand, at the inner boundary of the domain (the solar surface), we integrate the characteristic equations which are just another way to write the equations, allowing to define the boundary conditions not in terms of the primitive field quantities, but in terms of combination of their temporal derivatives. This corresponds to fixing the time variation of the fluctuations entering the numerical domain from below. The fields which intervene in the characteristic equations are the density $\rho$, velocity $u_{r}, u_{\theta}, u_{\phi}$, azimuthal magnetic field $B_{\phi}$, and the poloidal magnetic field $B_{\theta}$. Note that the radial magnetic field is absent from the list (the constraint $\operatorname{div} B=0$ is automatically taken into account). The poloidal magnetic field replaces the magnetic potential. Using Eq. (1), the solution of the characteristic equations at the boundary for $B_{\theta}$ and the solution for the potential $\psi$ are combined to extrapolate the potential at the boundary (see Grappin et al. 2000).

\subsection{Boundary conditions}

Only the in-going Alfvén characteristic is excited, the in-going slow and fast magneto-sonic waves are imposed to be zero. Hence, no pressure variation is introduced via the inner boundary: pressure variations at the inner boundary can only be the by-product of Alfvén wave interactions within the medium propagating back to the inner boundary. Exciting the Alfvén characteristic implies that we excite at the same time the azimuthal and the poloidal components of the velocity and magnetic field, so that the part of the magnetic pressure associated with the injected wave remains constant. Such a wave is a socalled arc wave (see Grappin et al. 2000). In practice however, the constancy of the magnetic intensity is lost: either during propagation of the arc wave in the stratified medium, or at the inner boundary because the upward propagating wave becomes mixed with waves propagating downward (GLH, Grappin et al. 2003a).

Note that, due to the axisymmetric conditions, no wave can be injected at the poles; also, no waves can be injected at the equator where the radial field goes to zero. In practice, injection is thus set to zero in small regions around the poles and equator (Grappin et al. 2003a, GLH).

It has been recently reported (Grappin et al. 2003b) that when injecting circularly polarized Alfvén waves at both footpoints of a coronal loop (within a simplified one-dimensional model), the resulting dynamics were largely determined by artificial coupling (reflections) at the boundaries. Some comments are necessary to explain why the situation is different here, and why the spurious coupling does not occur. In Grappin et al. (2003b), all fields depend on a single coordinate (the abscissa along the loop, the curvature being neglected), and one assumes a uniform magnetic field. To excite the circularly polarized wave, one uses two incoming characteristics: the fast and the Alfvén characteristics, the former being necessary to increase the magnetic pressure when starting the wave, and also when the magnetic intensity varies, once the wave arriving from the other foot-point becomes superposed on the injected one. It happens that in this latter case (superposition of both waves), the fast characteristic generates a persistent over-pressure which generates the siphon flow. In the present 2D simulations on the contrary, the incoming characteristics (fast or slow) that might directly inject pressure changes are not used: only the Alfvén characteristic is used. Hence, 
pressure variations can still occur but only as a byproduct of the dynamics acting inside the domain. In particular, pressure variations at the foot-points of the inner boundary will be triggered only by pressure fluctuations generated inside the domain and propagating downward towards the boundaries.

\subsection{Spatial scheme}

We use a modified compact finite difference spatial scheme (Lele 1992) as in GLH. Compact schemes have the property of giving an error which, for a given order, is much smaller than the corresponding explicit spatial scheme (see Lele 1992). These compact schemes become unstable when used with a nonuniform mesh via a coordinate mapping. However, this unstable behavior disappears when integrating the metrics, as we do here, in the definition of the spatial scheme (Le Saout 2003; see also Gamet et al. 1999).

The irregular grid is defined as follows. If $i$ is the index of the mesh point and $N_{x}$ the number of grid points, the heliocentric distance $r$ is defined as: $r(i)=r_{0}+\left(q^{i-1}-1\right) /\left(q^{N_{x}-1}-1\right) \delta R$ with $i$ running from 1 to $N_{x}, r_{0}=1 R_{\mathrm{s}}$ and $\delta R=15 R_{\mathrm{s}}$ and $q=1.01$.

This choice allows us to concentrate the resolution in the region of interest here, namely between 1 and 4 solar radii, which contains half the grid points. Another advantage is that it suppresses entirely a jump in current density which occurred at the boundary in the previous work with uniform mesh in GLH. This considerably improves the stability of the physical quantities in the vicinity of the inner boundary.

Dissipation at the grid scale is achieved by two different means: a filter, which is applied at each time step, and diffusive, Laplacian-like terms. The Laplacian-like term is the sum of a second derivative with respect to radial distance and of a second derivative with respect to latitude, with weights chosen so that the diffusion rate is adjusted to the local mesh size. No Laplacian is used for magnetic field, and a second derivative in latitude only is used for density, radial and poloidal velocity. A second derivative in radial distance is used for radial and poloidal velocity, except within a band of 50 radial mesh points close to the inner boundary. The filter is a 6th order filter (Lele 1992); it is applied independently in both $R$ and $\theta$ directions. Filtering in latitude is used for density in a band of 10 radial mesh points close to the inner boundary. Velocity, magnetic potential and the azimuthal component of magnetic field are filtered in both directions everywhere. Note that the solenoidal character of the magnetic field is not destroyed by using this dissipation scheme, since it is applied directly to the magnetic potential $\psi$, and not to the radial and poloidal components.

For a given resolution, this scheme leads to decreased dissipation of waves and flows close to the inner boundary, and on the contrary to increased dissipation at large distances. This is not a real drawback, since we are not really interested in this paper in the open wind region. The only reason why the supersonic wind region is present in the numerical domain is that it is required for physical and numerical stability as soon as there is a sonic point, that is, as soon as the decrease of gravitational force with distance is taken into account (Velli 1994).

\subsection{Physical and numerical parameters}

The distance interval corresponds to heliocentric distances between unity up to 16 solar radii but, as explained above, the resolution is concentrated between 1 and 4 solar radii. The number of grid points in both the radial and the azimuthal directions is $N_{x}=256$.

The coronal temperature is taken to be isothermal, equal to $1.3 \mathrm{MK}$. The corresponding sound speed (isothermal) is $146.5 \mathrm{~km} \mathrm{~s}^{-1}$. The initial state of the coronal base, (before the injection of waves begins) is characterized at the equator by a density which is $n=10^{8} \mathrm{~cm}^{-3}$, a magnetic field which is $2.74 \mathrm{G}$. The plasma $\beta$ is 0.03 at the poles, 0.04 at the boundary of the closed region ( $\pm 40^{\circ}$ latitude), and 0.12 at the equator. Note that, since only incoming fluctuations are controlled at the coronal base, these figures are the output of the computations; in particular, the magnetic field results from the addition of the given external field and of the induced field.

The main wave period is $T_{0}=18 \mathrm{~min}$, which in numerical units is $T_{0}=0.035$, the numerical time unit being $\tau=8.4 \mathrm{~h}$ (Table 1 gives the angular frequencies $\omega$ in numerical units). The amplitude of the wave injected at the inner boundary is $u_{\phi}=50 \mathrm{~km} \mathrm{~s}^{-1}$, except for the two runs Ra and S1a where the amplitude is $25 \mathrm{~km} \mathrm{~s}^{-1}$ (see Table 1). Additive interference with downward propagating waves raises the amplitude to about twice the injected amplitude in part of the closed region. The relative amplitude is $u_{\phi} / c_{\mathrm{a}} \simeq 0.06$ in the closed region, decreasing to 0.03 in open regions. These amplitudes are admittedly very large. However, we will see that relevant quantities such as density fluctuations and siphon velocities resulting from the wave injection scale as the square of the Alfvén wave amplitude. This scaling allows to easy generalization of the results to more realistic amplitudes.

Table 1 gives the list of runs. In all runs except run $\mathrm{R}$ the regions of excitation are symmetric with respect to the equator. In run $\mathrm{R}$, we excite only the south hemisphere. In runs $\mathrm{R}$ and $\mathrm{S} 1, \mathrm{~S} 2$, the excitation is extended to the whole meridian (S1 and S2) or a half meridian. In runs $\mathrm{T}$ and $\mathrm{U}$, one excites localized, small latitude intervals. In runs $\mathrm{R}, \mathrm{Sn}$ and $\mathrm{Tn}$, the excitation is monochromatic.

\section{Results}

We start with a quasi-stationary wind, basically that of Pneuman \& Kopp (1971), see also (GLH). The wind blows freely at high latitudes along magnetic field lines which are close to radial (there is no rotation). There is a stagnant streamer belt around the equator, where magnetic lines remain closed. These initial conditions are shown in Fig. 1, in a closeup around the streamer belt, in a radial distance-colatitude $(R-\theta)$ representation. The top panel shows the initial velocity field together with the plasma $\beta$ contours at $0.05,0.075$, 0.1 and 1 . Since the temperature is uniform, the contours reflect directly the strong stratification of the Alfvén speed, which will be shown later to have important consequences. Magnetic field lines are shown in the bottom panel: the boundary between open and closed field lines corresponds to the boundary 
Table 1. List of runs. "Injection range" indicates the colatitude range of the injection of Alfvén waves: "All" means whole meridian, "All South" means the south meridian. For runs T1 to U3, only the north colatitudes in degrees are indicated; actually, the interval symmetric with respect to equator is also excited; one has also mentioned the loop (A or B) closest to the interval (see Fig. 1). $\omega$ is the wave frequency (time unit is $8.4 \mathrm{~h}$, see text). When two frequencies are given (runs Un), the first one corresponds to the south foot-point, the other one to the north foot-point. "Delay" is the time delay between north and south excitation. "Siphon" indicates whether or not a siphon flow is obtained during a time interval $t=0.5$, its direction ( $\mathrm{S}-\mathrm{N}$ meaning south to north, $\mathrm{N}-\mathrm{S}$ the reverse), and eventually its location (if not indicated, siphon location is close to the excitation loop for runs Tn to Un). Amplitudes of the waves injected are all the same $\left( \pm 50 \mathrm{~km} \mathrm{~s}^{-1}\right)$, except for runs $\mathrm{Ra}$ and $\mathrm{S} 1 \mathrm{a}$ for which amplitude injected is $\pm 25 \mathrm{~km} \mathrm{~s}^{-1}$.

\begin{tabular}{ccccc}
\hline \hline Run & Injection range & $\omega$ & Delay & Siphon \\
\hline R & All South & 180 & NA & $\simeq$ loop C, S-N \\
Ra & All South (ampl. 1/2) & 180 & NA & $\simeq$ loop C, S-N \\
S1 & All & 180 & 0.1 & $\simeq$ loop A, N-S \\
S1a & All (ampl. 1/2) & 180 & 0.1 & $\simeq$ loop A, N-S \\
S2 & All & 180 & 0.15 & $\simeq$ loop A, S-N \\
T1 & {$[49.4,56.5] \geq$ loop B } & 180 & 0.1 & no \\
T2 & {$[60,67] \simeq$ loop A } & 180 & 0.1 & N-S \\
T3 & {$[67,74]<$ loop A } & 180 & 0.1 & no \\
T4 & {$[67,74]<$ loop A } & 220 & 0.1 & no \\
T5 & {$[67,74]<$ loop A } & 220 & 0.15 & S-N \\
U1 & {$[60,67] \simeq$ loop A } & 180,100 & 0.1 & no \\
U2 & {$[60,67] \simeq$ loop A } & 180,160 & 0.1 & no \\
U3 & {$[60,67] \simeq$ loop A } & $180,180 \pm 20$ & 0.1 & S-N \\
\hline & & & &
\end{tabular}

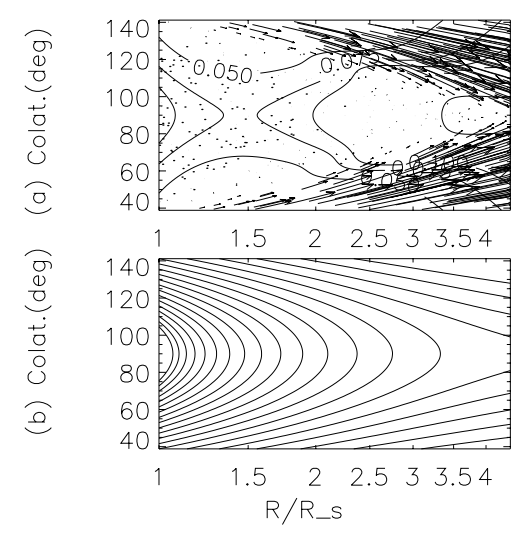

Fig. 1. $R-\theta$ map, close-up of the closed equatorial region (initial condition, $t=0$ ) showing the coronal streamer, radial heliocentric distances within $[1,4.4] R_{\mathrm{s}}$ and colatitude $\theta$ within $\left[40^{\circ}, 130^{\circ}\right]$. North is down, South is up. a) Velocity field, together with plasma $\beta$ contours; b) magnetic field lines.

between the flow (wind) region and the stagnant region visible in the top panel.

The simplest way to globally explore the response of the corona to Alfvén wave perturbations is to perturb all magnetic field lines. However, as mentioned in the introduction, different results are to be expected depending on whether single

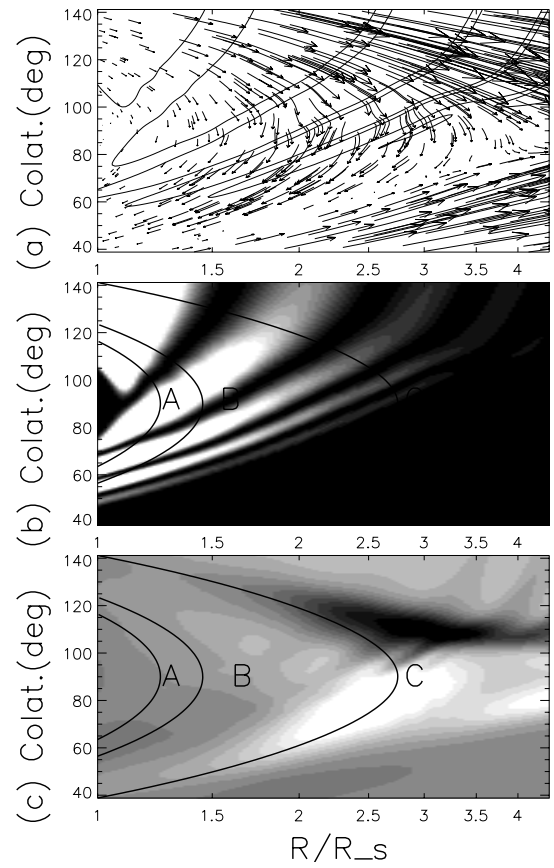

Fig. 2. Run R, $R-\theta$ map, close-up of the closed equatorial region at $t=0.5$ showing the coronal streamer, radial heliocentric distances within $[1,4.4] R_{\mathrm{s}}$ and colatitude $\theta$ within $\left[40^{\circ}, 130^{\circ}\right]$. North is down, South is up. From top to bottom: a) velocity field at $t=0.5$ (14 wave periods), together with contours of $u_{\phi}$ at $[-40,40] \mathrm{km} \mathrm{s}^{-1}$; b) three selected magnetic field lines, and image of magnetic wave pressure $B_{\phi}^{2}$ at $t=0.5$ (arbitrary units); c) same three selected magnetic field lines, and image of relative density increase $\delta \rho / \rho$ between time 0 and 0.5 : white is $30 \%$, black $-30 \%$.

foot-points or both foot-points are excited. We thus begin by exciting a single hemisphere, namely the south one.

\subsection{Injecting waves from one hemisphere}

Figure 2 shows the streamer region after injecting waves from the south hemisphere during about 14 wave periods $(t=$ $0.5)$. In panel (a), we have plotted the velocity field with $[-40,40] \mathrm{km} \mathrm{s}^{-1}$ contours of $u_{\phi}$. The two other panels show three selected magnetic field lines, on an image of the wave pressure, that is, $B_{\phi}^{2} / 2(\mathrm{~b})$, and on an image of the relative density change between time $t=0$ and time $t=0.5(\mathrm{c})$. Note that the three selected magnetic field lines shown in panels (b) and (c) are in fact representative of the initial configuration as well, since the perturbation is not large enough to perturb significantly the field lines.

After 14 periods, there is ample time for the wave pattern (Figs. 1a or 1b) to propagate all along the field lines: there are about two wavelengths along loop B, that is, two wave periods, and about four along the longest loop C. Hence, the apparent confinement of the wave in the south hemisphere (where the injection is made), visible in panels (a) and (b), cannot be due to the fact that the wave fronts did not reach the other hemisphere. The reason for this confinement must lie in the damping of waves at the diffusive scale, that is, the mesh size. This damping occurs because the wavelength, initially large close to the inner boundary is forced to decrease as it propagates, due to 

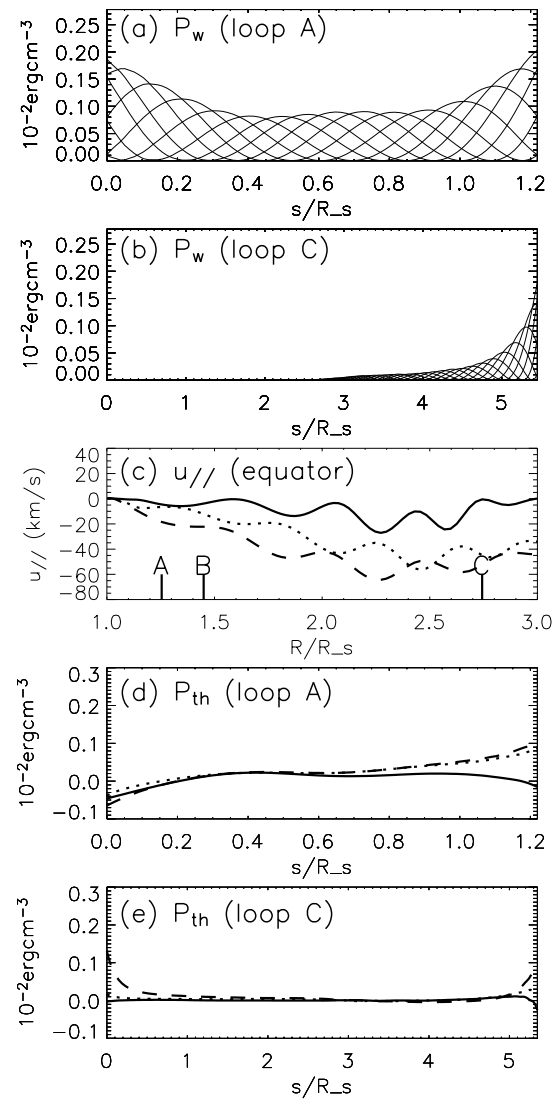

Fig. 3. Run R. a), b): wave pressure vs. position along loops A and C, one wave period; c): parallel velocity vs. heliocentric distance along equator, at times $t=0.1,0.3,0.5$ (resp. continuous, dotted, dashed). $\mathrm{A}, \mathrm{B}$ and $\mathrm{C}$ indicate the positions of the apex of the loops shown in Fig. 1. Negative velocity means northward flow; d), e): pressure difference vs. position along loops $\mathrm{A}$ and $\mathrm{C}$ at $t=0.1,0.3,0.5$. Abscissa starts at north foot-point, so that the excited foot-point is at right in panels a), b), d), e).

the large Alfvén speed gradients visible in Fig. 1a, which force the wavefronts to turn away from parallel to the surface as the wave propagates along the field lines. The mesh size, and thus the dissipation scale, is reached before the wave reaches the other loop end, for the longest loops at least.

The damping length being identical to the mesh size, one sees that the damping length depends on the local resolution. In other words, which loop shows damping and which loop shows no damping relies on the numerical setup, while the real effective damping length is many orders of magnitude smaller than any reachable mesh size. However, the qualitative result, that is, the fact that the longest loops should show damping, should be independent of the simulation setup. This point will be discussed again later.

The bottom panel of Fig. 2 shows that the density stratification within the streamer reflects the north-south dissymmetry of the wave profile along the longest loops: the density is enhanced in the northern branch close to the apex of the longest lines, while it decreases in the southern branch. The maximum enhancement at that time is $\delta \rho / \rho=47 \%$, the largest relative decrease is $34 \%$.
Figures $3 \mathrm{a}$,b show the wave pressure $B_{\phi}^{2} / 2$ along loops $\mathrm{A}$ and $\mathrm{C}$, sampled during one wave period at $t=0.5$. It again illustrates the difference between the short (A) and the long (C) loops. In principle, the long field lines $(\mathrm{C})$, along which the wave experiences strong damping, should exhibit strong acceleration of the plasma in the direction of wave propagation, along the field lines. Indeed, Fig. 2a shows that at $t=0.5$, inside the previously stagnant streamer belt, a circulation is directed northward, in the direction of wave propagation, roughly parallel to the field lines. The central panel (c) in Fig. 3 shows this in more detail: at the same time $t=0.5$ (dashed line), the parallel velocity, that is, the velocity projected on the magnetic field in the meridional plane, is largest at radial distances between 2.2 and 2.7 solar radii, which includes the apex of loop C. (Note that a negative velocity as shown corresponds to a northward circulation.)

However, the same Fig. $3 \mathrm{c}$ shows that the acceleration is not constant in time: it is weaker in average between $t=0.3$ and 0.5 than between $t=0$ and 0.3 . Since the average wave pressure has not changed (apart from a short transient), it must be that the thermal pressure has changed. To check this point, we plot in the two last panels of Fig. 3 the change in thermal pressure $\delta P_{\text {th }}=P_{\text {th }}(t)-P_{\text {th }}(0)$ along the two lines $\mathrm{A}$ and $\mathrm{C}$ at times $t=0.1,0.3,0.5$. We see that, for both loops, the change in thermal pressure $\delta P$ at time $t=0.5$ is comparable to the amplitude of the wave pressure (compare panels (d), (e) with panels (a), (b)). The pressure in loop A increases with time at the south foot-point and decreases at the north foot-point. The whole profile is almost stationary at $t=0.5$. This suggests that a south-north siphon flow could emerge at a later time in loop A. On the contrary, loop C shows an increase of pressure at both foot-points, thus moderating the wave pressure imbalance found in Figs. 3a,b: this explains the quasi-relaxation of the siphon flow seen in Fig. 3c.

To better quantify the respective contributions of the wave pressure and of the gas pressure change to the acceleration process would require evaluating the gradient of these quantities along the loops and dividing by the density, which is higher at the foot-points than at the apex of the loops (the maximum density contrast along loop $\mathrm{C}$ is about 400 ). Also, we should take into account the density change, that is, the buoyancy force along the loops. This will be done later on for run $\mathrm{S} 1$.

It is of some importance to note that the time scales necessary to achieve equilibrium vary, depending on the region we consider. As we are considering pressure variations along the magnetic field lines, the sound speed is relevant. This sound speed is assumed to be $146.5 \mathrm{~km} \mathrm{~s}^{-1}$, so that for loop A, whose length is about $1.21 R_{\mathrm{s}}$ (Fig. 3a), the sound propagation time is $\Delta t_{\mathrm{A}}=0.19$, a time substantially shorter than the time scale we have considered up to now $(\Delta t=0.5)$. On the contrary, the sound propagation time along loop $\mathrm{C}$, whose length is $5.4 R_{\mathrm{S}}$, is about 0.76 , which is noticeably larger than 0.5 . In line with these arguments, we find that the mass flux becomes rapidly about uniform along loop A, but not along loop $\mathrm{C}$, where only the south leg of the loop shows a uniform mass flux at time $t=0.5$ (dashed curve in Fig. 4b). The mass flux plotted in Fig. 4 is evaluated as $F=\rho u_{\|} A$, where the cross section A 


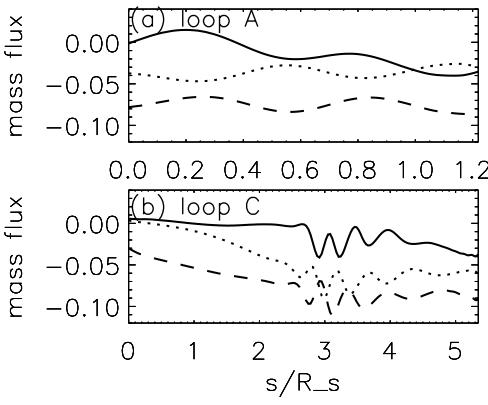

Fig. 4. Run R. Mass flux estimated as $F=\rho u_{\|} / B$ (see text) vs. position along loops A (a)) and C (b)) at $t=0.1,0.3,0.5$ (resp. continuous, dotted, dashed). Abscissa starts at north foot-point, so that the excited foot-point is at right in the figure.

is replaced by $1 / B$, using the conservation of magnetic flux, and $\left.B=\sqrt{(} B_{r}^{2}+B_{\theta}^{2}\right)$.

\subsection{Injecting waves from two hemispheres}

We consider now the effect of shaking both the north and south foot-points of magnetic loops, that is, exciting both hemispheres with Alfvén waves. We shake first the south hemisphere at time $t=0$, and the north hemisphere slightly later, at time $t=0.1$ (run S1). We have checked that only the phase difference matters, not the difference in starting times. The frequency is the same as in run $\mathrm{R}$ (period $\tau=.035$, that is, $18 \mathrm{~min}$ ). Figure 4 shows for run $\mathrm{S} 1$ the streamer region at time $t=0.5$, in the same way as Fig. 2 for run R. The top panel shows the velocity field, together with the $[-40,40] \mathrm{km} \mathrm{s}^{-1}$ contours of azimuthal velocity. The mid panel shows the three magnetic field lines already considered in Fig. 2, together with an image of the wave pressure $B_{\phi}^{2} / 2$. The bottom panel shows again the three magnetic field lines, with an image of the relative density change between time 0 and 0.5 .

One can distinguish two regions in panels (b) and (c): the open region, south and north, where waves are progressive, that is quasi-plane waves with wave fronts tilted and distorted due to gradients of Alfvén speed, and the closed region where interference between the two waves propagating in opposite directions along field lines is apparent. Note that, when viewed in the twodimensional $R-\theta$ plane, the interfering wave pattern is actually drifting outwards, the propagation speed depending on the relation between the apex height and the loop length. However, the interference region appears clearly smaller than the closed region, in particular it is smaller than the region enclosed within loop C. This is due to the dissipation of the wave at the mesh size induced by the tilting of the wave fronts and subsequent decrease of the wavelength, as was the case in run R.

As shown in the top panel, the flow in the closed region at time $t=0.5$ adopts a configuration that is rather different from that of the previous run $\mathrm{R}$ where only one hemisphere is excited. The longest closed loops do show substantial parallel flows but, contrary to run R, no dominant circulation arises there: the flow is directed outward, towards the apex of the loops. This does not correspond to a stationary regime, as will be confirmed below. On the other hand, there is now a systematic north-south circulation, that is, reverse of that observed in run $\mathrm{R}$, along the shortest excited loops, around loop A.

Let us examine finally the relative change in density between $t=0$ and $t=0.5$, shown in the bottom panel. Two regions of density enhancements are visible: the interference region on the one hand, and the heliospheric current sheet, on the other hand. The density enhancement in the latter region was already mentioned in GLH, and explained to be due to the snow-plough effect of the fast wind, north and south, impinging on the equatorial region. We concentrate here on the interference region. The interference region shows radial "fingers" of enhanced density, while the end of the streamer shows actually the highest relative density enhancement (note that, white and black in the image are associated with $\pm 30 \%$, while the real extrema are -0.31 and 1.2 ). When comparing panels (b) and (c), one sees that the density "fingers" show up precisely in the radial lows of the wave pressure pattern, which are timeindependent, in spite of the uniform outward radial drift of the pattern already mentioned. The important point is that the new density pattern is quasi-stationary.

Let us concentrate on loops $\mathrm{A}$ and $\mathrm{C}$, and consider first the wave pressure pattern (Figs. 6a,b). Along loop C, the two progressive waves are damped before they are able to interfere; the wave pattern is thus completely symmetric with respect to the equator (where the loop apex lies), and there is no reason to expect a significant break in the pressure balance between the two foot-points. This explains why no substantial circulation appears along loop C. In the much shorter loop A on the contrary, damping can be neglected, so that the two progressive waves interfere fully: the wave pressure shows standing oscillations, with large amplitude disequilibrium between the two foot-points, as the south foot-point is located at a zero of the interference pattern (Fig. 6a).

On average, the wave pressure pattern along loop A, as shown in Fig. 6a, should have two effects: it should push the plasma within the pressure troughs, which should modify the gas pressure field along the loop: this explains the "fingers" in Fig. 5c. The resulting total (wave + gas) pressure pattern could in turn trigger the siphon flow observed in Fig. 5a. To investigate this point, we plot in Fig. $6 \mathrm{c}$ the variation of the gas pressure field $\delta P_{\text {th }}=P_{\text {th }}(t)-P_{\text {th }}(0)$, along loop A, at times $t=0.02, \ldots 0.5$, to see its evolution. As in run $\mathrm{R}$, the amplitude of the pressure change is again rapidly comparable to that of the wave pressure: the new pressure field stabilizes around $t=0.25$. Since the wave pressure becomes stationary even sooner, we infer that the global acceleration, if any, due to the contribution of both the wave pressure and the new pressure field, should be constant after time $t=0.25$.

The situation for run $\mathrm{S} 1$ thus differs from run $\mathrm{R}$ in that the wave and gas pressure combine to trigger a siphon flow, and that this occurs in the region of short loops. To assess this point, we evaluate the gradients in more detail. This is done in Fig. 7, which shows the contribution of the wave pressure (a), and (b) the sum of the contributions of wave and thermal pressures, subtracting the hydrostatic contribution. From this figure, one can conclude that, if the acceleration were comparable everywhere along the loop to one of its peak value (say, 


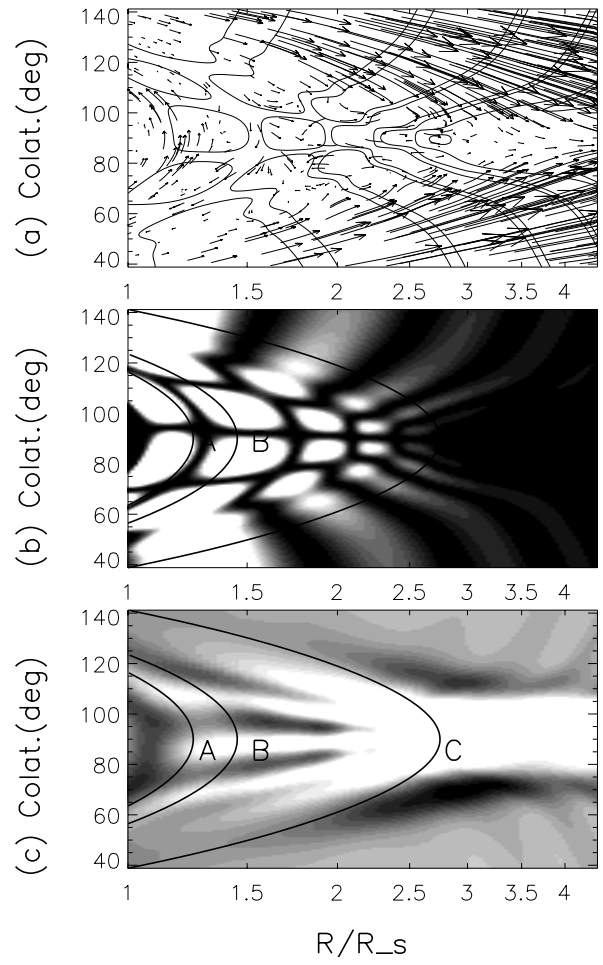

Fig. 5. Run $\mathrm{S} 1, R-\theta$ map, close up of the closed equatorial region as in Fig. 2 at $t=0.5$. From top to bottom: a) velocity field, together with contours of $u_{\phi}$ at $[-40,40] \mathrm{km} \mathrm{s}^{-1}$; b) three selected magnetic field lines (same as Fig. 1), and image of magnetic wave pressure $B_{\phi}^{2} / 2$; c) three selected magnetic field lines and image of relative density increase $\delta \rho / \rho$ between time 0 and 0.5 : white is larger or equal to $30 \%$, black is $-30 \%$. (the actual extremal values in the domain are larger).

$50 \mathrm{~m} / \mathrm{s}^{2}$ ), the parallel flow would reach a speed of $10 \mathrm{~km} \mathrm{~s}^{-1}$ in about $200 \mathrm{~s}$, that is, in a small fraction of the Alfvén wave period. However, the true acceleration process is actually much slower than that, because of the numerous sign reversals of the acceleration along the loop. One sees that only the total acceleration shows, on average, a clear-cut definite positive sign, indicating a mean southward acceleration, corresponding to the observed circulation. Note however that this diagnostic is not fully complete, as it neglects curvature terms in the expression of the wave pressure, which actually contribute to the parallel Lorentz force. Also, the wiggles visible in Fig. $7 \mathrm{~b}$ are due to the data analysis being not refined enough: they come from computing the derivatives of fields after interpolating along the loop. In contrast, derivatives computed without interpolation and during the numerical integration do not show such wiggles.

Are there other loops, apart from loop A and immediate neighbors, along which a mean acceleration with a definite sign can be found? One expects to find a mean acceleration only in the interference region, since elsewhere the average pressure (both gas and wave) patterns will be symmetric in the south and north hemispheres, at least as long as amplitudes injected are equal in north and south foot-points. Also, for a given loop, the pressure disequilibrium will depend on the phase of the interference pattern along the loop, and on the resulting gas pressure field. Since the interference pattern along the loop in principle varies with the length of the loop, one would expect
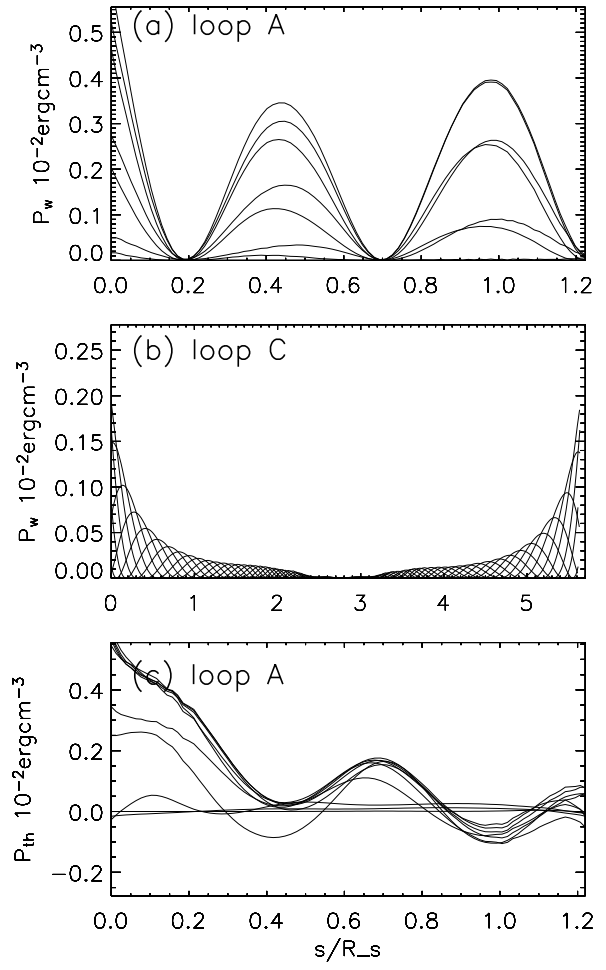

Fig. 6. Run S1. Wave pressure vs. position along loops A (a)) and $\mathrm{C}(\mathbf{b})$ ), during one wave period; $\mathbf{c})$ : gas pressure variation vs. position along loop A, $t=0.02, \ldots 0.5$.
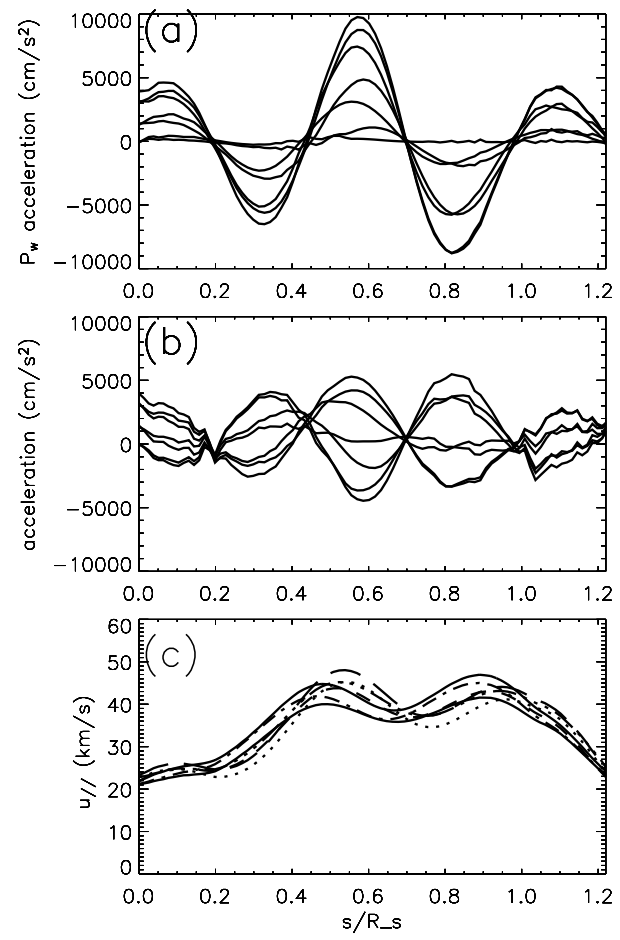

Fig. 7. Run S1, sample profiles vs. position along loop A, during one wave period around $t=0.5$. a) Acceleration $-P_{\mathrm{w}}^{\prime} / \rho$ due to wave pressure $\left.P_{\mathrm{w}} ; \mathbf{b}\right)$ acceleration due to wave pressure and thermal pressure: $-P_{\mathrm{w}}^{\prime} / \rho-P^{\prime} / \rho+\left(P^{\prime} / \rho\right)(t=0)$ (prime denotes derivative with respect to abscissa along loop); c) parallel velocity. Positive parallel velocity or acceleration means southward. Abscissa starts at the north foot-point, so that the excited foot-point is to the right. 

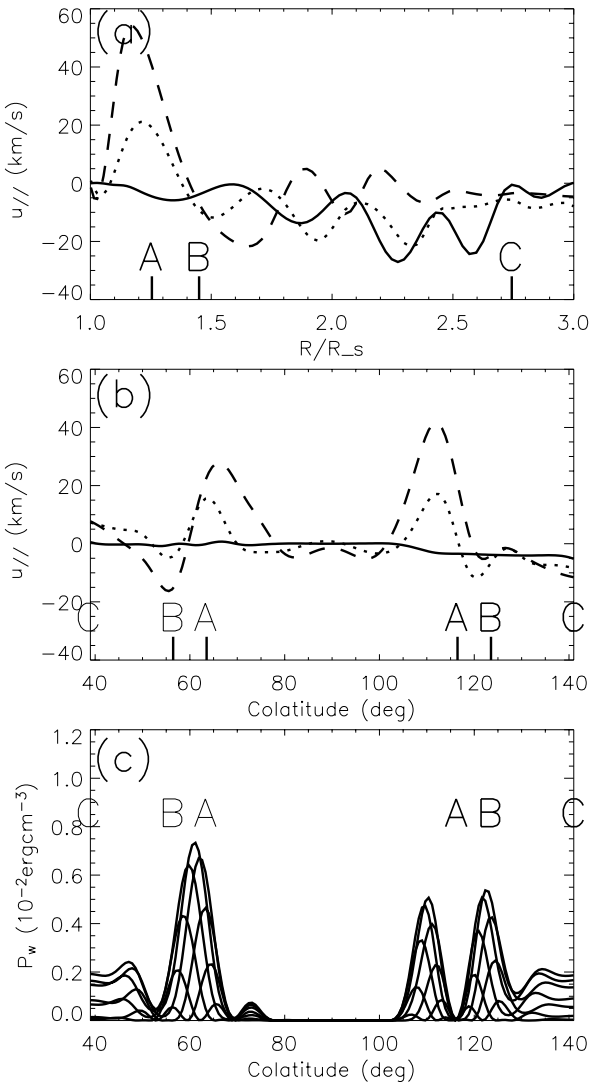

Fig. 8. Run S1. a), b): parallel velocity vs. radial distance along equator and vs. colatitude at inner boundary, at times $t=0.1,0.3,0.5$ (resp. continuous, dotted, dashed lines); c): wave pressure vs. colatitude at inner boundary. Loops A, B and C are marked for their apex in a) and footpoints in b) and c).

to find several sign reversals of the average acceleration when considering loops with increasing lengths, hence possibly several siphon flows with opposite directions (north-south or reverse). However, visual inspection of Fig. 5 does not reveal any siphon flow other than the one already found around loop A.

To assess this point, we plot in Figs. 8a,b the velocity parallel to the magnetic field, along two directions: along the equator, versus radial distance, and along the inner boundary, versus colatitude. We show profiles at three times, $t=0.1,0.3,0.5$. We have plotted for comparison in Fig. 8c the wave pressure vs. colatitude at the inner boundary, during one wave period. A siphon flow would be revealed by a persistent peak with a given sign in the top panel, and by double peaks of the same sign in the mid panel, with latitudes corresponding to foot-point pairs. All these conditions are met for loop A, with a positive parallel velocity, that is, southward. For loop A, one also sees that the average acceleration is almost constant, when comparing the variations during the two time intervals $[0.1,0.3]$ and $[0.3,0.5]$. This is expected from the steadiness of the wave pattern and pressure pattern. A secondary siphon flow, with reverse direction (south-north), is visible around loop B. However, its peak is less pronounced, its position changes with time, and its growth is much slower. At larger distances, one finds as expected no definite sign for the velocity, as we are outside the interference region.

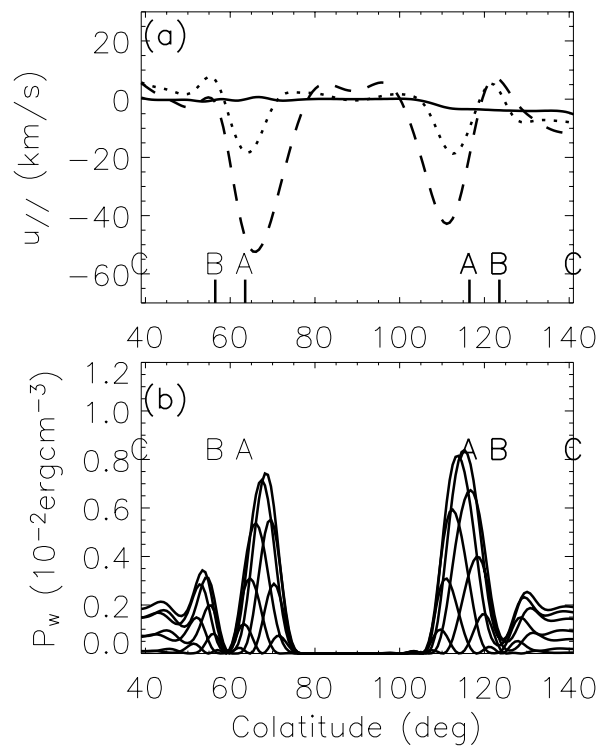

Fig. 9. Run S2. a) Parallel velocity vs. colatitude at inner boundary at times $t=0.1,0.3,0.5$ (resp. continuous, dotted, dashed lines); b) wave pressure $B_{\phi}^{2} / 2$ vs. colatitude at inner boundary versus colatitude, several profiles during one wave period $(t=0.5)$. Foot-points latitude of loops $\mathrm{A}, \mathrm{B}$ and $\mathrm{C}$ are indicated.

Are these results changed if we change the phase delay between the north and south hemispheres? Figure 9 shows results obtained with the time delay $\Delta t=0.15$ (run S2). The figure shows the parallel velocity at $t=0.1,0.3,0.5$ (top) and the wave pressure pattern (bottom), during one wave period around $t=0.5$, at the inner boundary. One sees that the wave pressure pattern is similar to run S1, but with two main peaks instead of three. There is a siphon flow again centered on loop A, but now in the south-north direction (compare with Figs. 8b,c). This reversal is not particularly surprising. Coming back to the case of run $S 1$, we see by examining Figs. $6 a$ and $7 b$ that a spatial shift in the wave pressure pattern along the loop (which would result from a shift in the phase difference between the two waves) can easily induce a change in the average sign of the acceleration profile (Fig. 7b), and thus finally in the direction of the flow.

\subsection{Selecting flux tubes: Location, phase, frequency}

The preceding section has shown the basic role of the moving interference pattern for the building of siphon flows. Injection of Alfvén waves was realized coherently in each hemisphere, which is perhaps not realistic, and also might hide how the coupling occurs between waves and flows. Since the issue of single foot-point excitation is reasonably clear, we concentrate on double foot-point excitation. A first point is whether the only region which can give rise to a siphon flow is around loop A, and whether local excitation works as global excitation does or not. This is related to the question of the optimal phase difference between the two loop ends, and its relation to the loop length. Our last point is related to the excitation frequencies. It is not evident that the phenomenon will persist when the two footpoint frequencies differ, or vary. 
The absence of clear multiple siphon flow structure is related to the large size (in latitude) of the magnetic pressure peaks in runs $\mathrm{S} 1$ and $\mathrm{S} 2$, a size comparable to that of the interference region (Figs. 8c and 9b), where large deviations from pressure balance between foot-points is possible. A detailed discussion of this point would need to relate propagation time and latitude in the closed loop system. Roughly speaking, to obtain smaller sizes, and hence possible multiple siphon systems, would require smaller wavelengths, that is in practice higher resolutions. Nevertheless, the absence of multiple siphon flows in the present simulations probably means that the conditions to be met to obtain a siphon flow are rather restrictive: only a small set of loops and/or parameter values are favorable.

To check this point, we selectively excite pairs of footpoints of thin flux tubes, that is, limited latitude intervals, keeping the same frequency and phase delay between the south and north foot-point as in run $\mathrm{S} 1$. In run $\mathrm{T} 1$ we excite a group of loops longer or equal to loop $\mathrm{B}$, namely the interval $\left[49.4^{\circ}, 56.5^{\circ}\right]$ (together with the symmetric interval in the other hemisphere). This latitude interval is a subset of the interference region; see for instance Fig. 9b for comparison with run $\mathrm{S} 2$. We found no siphon flow. In run $\mathrm{T} 2$, the excitation is centered on loop A (in $\left[60^{\circ}, 67.1^{\circ}\right]$ ). This run shows a siphon flow (Fig. 9), with the same direction as the siphon flow of run $\mathrm{S} 1$. Figure 10 shows from top to bottom, at $t=0.5$, azimuthal velocity contours with the three loops A, B, C; again the azimuthal velocity contours with the velocity field, and the relative density increase between $t=0$ and $t=0.5$. One sees that the excited loop system is traversed by a northward siphon flow, and that a large part of the loop system appears to be over-dense, the maximum over-density being $14 \%$. Note that all quantities shown occupy a similar region in the $R-\theta$ plane. However, as time goes on, the region showing significant parallel velocity and over-density progressively increases, invading a larger and larger part of the coronal streamer.

We then investigate whether loops shorter than loop A could also show a siphon flow. We tried without success the interval $\left[67.1^{\circ}, 74.1^{\circ}\right]$ (run T3). Then we changed the frequency to $\omega=220$, with the same negative result (run T4). Only when we changed the time delay to $\Delta t=0.15$ (run T5) did we finally recover a siphon flow, with direction south-north as in run S2.

What happens when considering different frequencies for exciting the south and north foot-points of a loop system? We expect in that case that the wave pressure, instead of adopting a standing oscillation pattern as in Figs. 5b and 6a, will drift along the loop (instead of just drifting away in the radial direction). This might prevent the onset of a constant gas pressure pattern as in previous runs, hence precluding the onset of an acceleration field with given direction along the loop. This would possibly suppress the siphon onset.

To examine this point, we consider in turn the three runs $\mathrm{U} 1, \mathrm{U} 2$ and $\mathrm{U} 3$, all with a narrow excitation region as in run $\mathrm{T} 2$, that is, around loop A. In runs $\mathrm{U} 1$ and $\mathrm{U} 2$, the south and north frequencies differ by $44 \%$ and $11 \%$ respectively (see Table 1). In both cases, no siphon flow appears. However, this does not prove that one could not find an appropriate phase

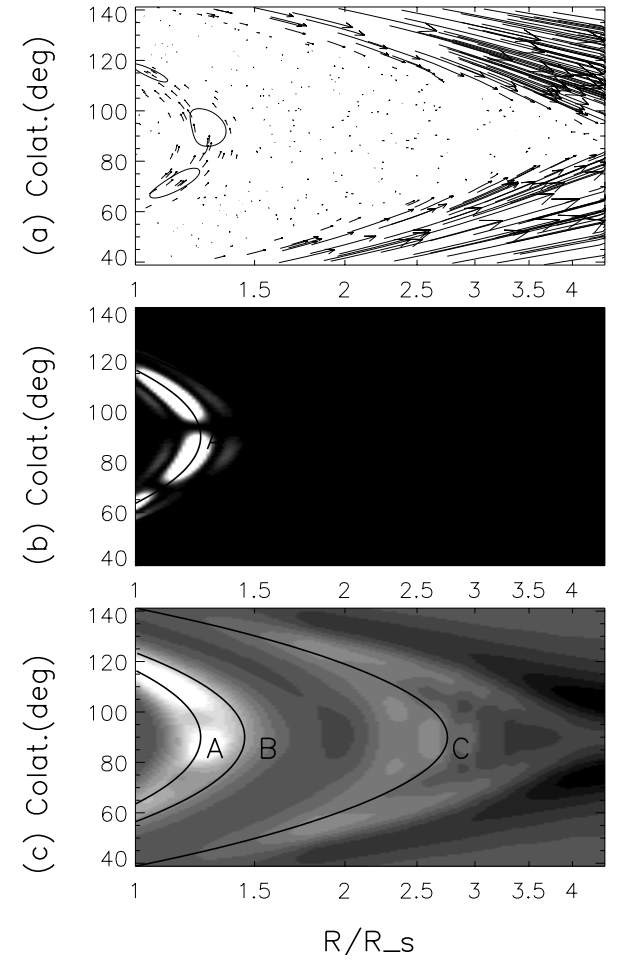

Fig. 10. Run T2, $R-\theta$ map, close up of the closed equatorial region as in Fig. 4. From top to bottom: a) velocity field, together with contours of $u_{\phi}$ at $\left.[-40,40] \mathrm{km} \mathrm{s}^{-1} ; \mathbf{b}\right)$ three selected magnetic field lines, and image of magnetic wave pressure $B_{\phi}^{2} / 2 ; \mathbf{c}$ ) three selected magnetic field lines and image of relative density increase $\delta \rho / \rho$ between time 0 and 0.5 : white is larger or equal to $10 \%$, black is $-10 \%$.

difference between north and south excitation which would trigger a siphon flow.

A more interesting (final) test is provided by run $\mathrm{U} 3$, in which we vary continuously this phase difference, or the frequency, which is equivalent. The south excitation is monochromatic $(\omega=180)$, but the north excitation has a frequency varying with time around 180 , with amplitude \pm 20 . Figures $11 \mathrm{a}, \mathrm{b}$ allow to compare the parallel velocity evolution for runs T2 and U3 along loop A. Profiles are shown at times 0.1,0.3 and 0.5: the final peak parallel velocity is substantially larger in run $\mathrm{U} 3$ than in run $\mathrm{T} 2$.

A more detailed comparison is shown in Fig. 12. The two upper panels show the temporal evolution of the parallel velocity at the apex of loop A for runs T2 and U3. One sees comparable evolutions up to $t=0.5$ (albeit with reverse velocities). There is first a flat curve during which a very weak (northward) acceleration is observed: this corresponds to the period during which only the south foot-points are excited, plus the Alfvén transit time of the northward excitation up to the apex of the loop. After that, there is a monotonous growth of the parallel velocity, that is, a constant southward acceleration, with an abrupt increase at the end of the period for run U3. Continuing run U3 up to $t=2$, shows (panel (c)) that on a larger time scale, the average acceleration is comparable for runs $\mathrm{T} 2$ and $\mathrm{U} 3$, in spite of irregularities in run $\mathrm{U} 3$, with a periodicity roughly equal to that of the frequency modulation of the north foot-point excitation, showing that the flow answers 


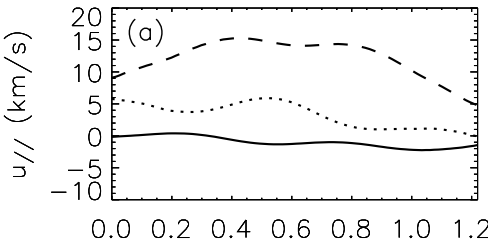

s/R_s

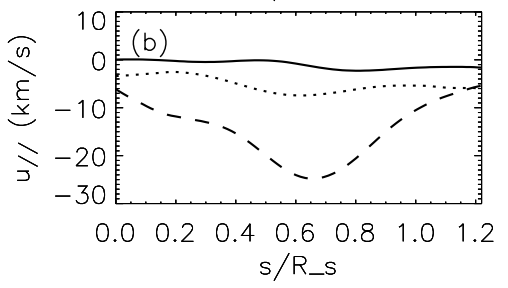

Fig. 11. Runs $\mathrm{T} 2$ a) and $\mathrm{U} 3$ b). Parallel velocity vs. position along loop A, at times $t=0.1,0.3,0.5$ (resp. continuous, dotted, dashed lines).

the periodic frequency modulation by a periodically "resonant" acceleration.

\section{Discussion}

We explored how Alfvén waves injected in an isothermal corona can trigger the growth of siphon flows in the excited loops, assuming transparent boundary conditions. The main finding of this work is that the Alfvén wave pressure induces a non trivial modification of the gas pressure stratification along the excited loop (or the loop ensemble), which in turn, together with the Alfvén wave pressure, determines the dynamic evolution of the plasma. The balance between the modified gas pressure, wave pressure and gravity is slowly, or never achieved, which makes possible the appearance of siphon flows. We considered two modes of excitation: single foot-point excitation and double (south and north) foot-point excitation.

In the case of single foot-point excitation, only the longest loops (that is, long enough for the wave to dampen significantly) give rise to persistent parallel flows. The parallel acceleration stops or at least decreases after some time, because the wave pressure modifies the pressure field so as to approximately balance the wave pressure gradients. Hence, asymptotic equilibrium can be achieved. Although the numerical damping in our simulation is certainly over-estimated compared to the coronal one, we think that the result is nevertheless relevant, since even with a much smaller damping rate, loops with foot-points close to the streamer boundary should lead to damping because Alfvén speed gradients are very high there. Note that the wave damping associated with Alfvén speed inhomogeneities is usually studied in the framework of non selfconsistent magnetic field structures, while in the present work the whole magnetic structure, and the Alfvén speed gradients as well, are consistently linked to the existence of a global (potential) field produced by the Sun, and to the induced field produced by the wind and the quasi-stationary closed structure imprisoned around the equator.

Siphon flows appearing when both foot-points are excited with the same amplitude differ in two respects from the previous ones: no equilibrium seems possible, and siphon flows
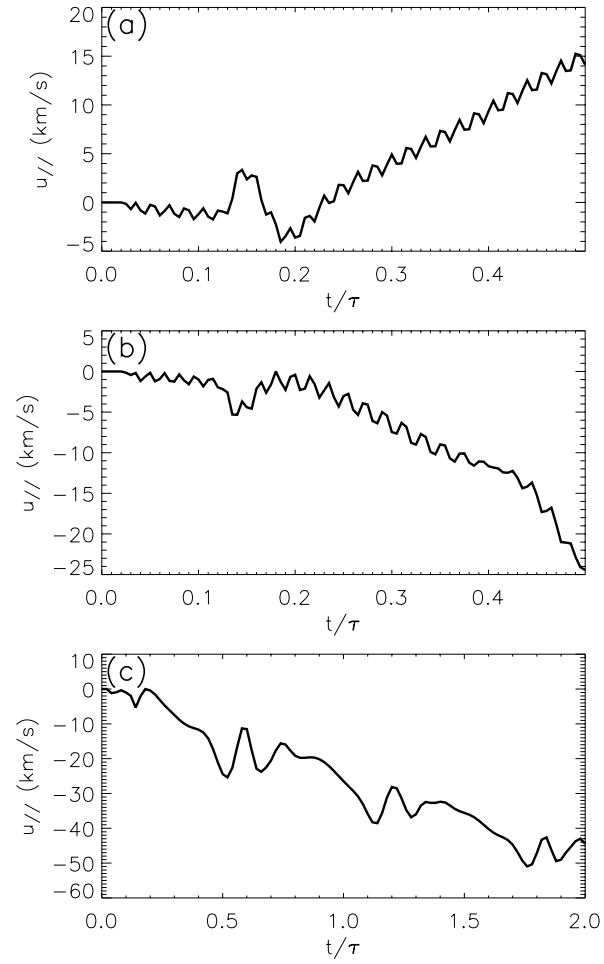

Fig. 12. Parallel velocity vs. time, at apex of loop A for runs $T 2$ a) and $\mathrm{U} 3$ b) and c). a) Short time evolution, run T2; b) short time evolution, run U3; c) long time evolution, run U3. Time is given in numerical unit $\tau=8.4 \mathrm{~h}$.

form (at least initially) in short loops, where dissipation can be neglected, and where the interference pattern is the most contrasted. It is interesting to compare the present 2D situation with the one-dimensional situation, with boundary conditions appropriate to ensure real transparency (Grappin et al. $2003 \mathrm{~b}$ ). In the $1 \mathrm{D}$ case, the interference of two circularly polarized Alfvén waves injected at both loop ends leads to a static spatial modulation of the magnetic pressure. The plasma being pushed within the troughs of the magnetic pressure pattern, total pressure balance is rapidly achieved, once all propagative compressive perturbations have left the loop. In the present 2D case on the contrary, a spatial modulation of the magnetic pressure also appears in the interference region, but the whole wave pressure pattern shows a permanent outward radial drift, so that, although a new quasi-stationary gas pressure pattern along the loop rapidly emerges as in the $1 \mathrm{D}$ case, the resulting total (wave plus gas) pressure is not able to achieve equilibrium. This absence of strict equilibrium does not automatically lead to a siphon flow, but makes it possible. Depending on the phase difference between the wave injected at the south and north loop ends, the total pressure stratification in the south and north loop legs will be more or less favorable for the growth of a siphon flow. In the long term, the region with siphon flow grows and progressively fills the whole closed region, so that this regime leads to global destabilization of the heliospheric current sheet and to the disappearance of the slow solar wind (GLH, Grappin et al. 2003a).

The acceleration of the siphon flow is related to the existence of a quasi-stationary interference wave pattern of the 


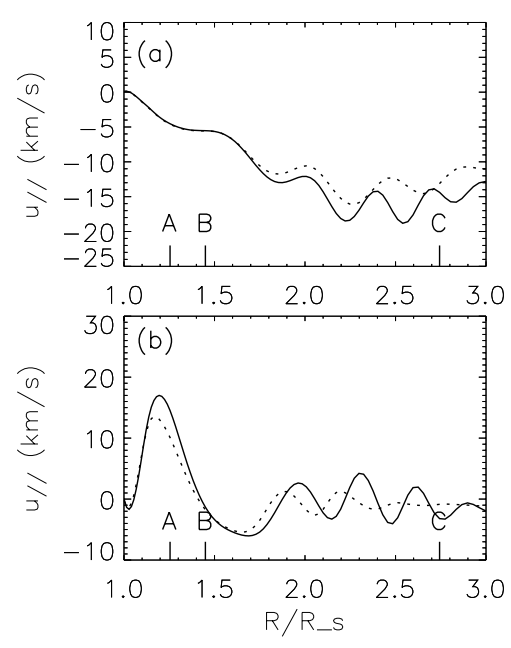

Fig. 13. Parallel velocity at $t=0.5$ vs. radial distance along equator, scaling with wave amplitude. a) Continuous line: parallel velocity in run $\mathrm{Ra}$; dotted: parallel velocity in run $\mathrm{R}$, multiplied by $1 / 4$; $\mathbf{b}$ ) continuous line: parallel velocity in run S1a; dotted: parallel velocity in run $\mathrm{S} 1$, multiplied by $1 / 4$.

magnetic wave pressure. A clear indication that the ponderomotive force is responsible for the acceleration is provided by the scaling of siphon speed amplitude (and amplitude of relative density fluctuations as well) with the square of the wave amplitude. Figure 13 compares parallel velocities along the equator for the two pairs of runs, $\mathrm{R}$ and $\mathrm{Ra}$ on the one hand, S1 and S1a on the other hand. Each pair differs by the amplitude of the Alfvén wave injected. In each panel, we have renormalized velocities for runs R and S1 to show the scaling. One sees that the scaling with the square of the wave amplitude works, in the range where siphon flows appear, that is, a very large region for runs $\mathrm{R}$ and $\mathrm{Ra}$, and a region close to loop $\mathrm{A}$ for runs $\mathrm{S} 1$ and S1a.

One might doubt that the siphon flow phenomenon still occurs when the north and south foot-point frequencies are not equal (runs Un). Indeed, in that case, there is a drift of the wave interference pattern along the loop, which should in principle prevent the formation of a long-term persistent disymmetry between foot-points as observed in the case with equal frequencies. Indeed, we could not find a siphon flow when adopting a $20 \%$ difference between the two exciting frequencies. However, in the more general case of a time-varying frequency for one of the two foot-points (run U3), which may be viewed as a proxy for a rich spectrum, with common frequencies between the two foot-points, we recovered a siphon flow. In this case, the siphon shows successive acceleration phases when resonance occurs, that is, each time the drift of the interference pattern along the loop becomes slow enough.

Other plasma properties which result from Alfvén wave excitation are the radial drift of the interference pattern and density fluctuations. The wave pressure pattern (and more generally the pressure pattern) drifts at a very large phase velocity (which may be much larger than the Alfvén speed) along the radial direction. The phase speed (compared with Alfvén speed) is determined by the relation between the length and the height of the loops, that is, the aspect ratio of the loops.
If the wave pressure pattern would be observable, one would thus find abnormally high phase speeds, which would be related to the geometry of the loops, A more important property, directly observable, is the modification of the plasma density, which occurs whether siphon flows develop or not. This is due essentially to the fact that the conditions of propagation are rarely such that Alfvén waves can retain ideal properties (constant magnetic pressure). In general, a permanent increase of the density is generated, with possibly additional density oscillations. Both kinds of density perturbations (the steady and the oscillating), have their amplitude scaling as the square of the injected Alfvén wave. Oscillations show a period which is here $10 \mathrm{~min}$, half that of the mother Alfvén wave, corresponding to this scaling. Figure 14 summarizes the different possibilities reported here in the different runs. It gives density vs. latitude at two heliocentric distances, namely 1.25 and $2.74 R_{\mathrm{s}}$, that is, respectively at the apex of loop $\mathrm{A}$, and the apex of loop $\mathrm{C}$, during one wave period, at $t=0.5$, with the initial profile added (dotted line). In the case of excitation by a single foot-point ((a), (b)), one observes a 5\% increase of density at short distances. At long distance, there is an increase or a decrease, depending on whether one considers the emission side (south), or the north side. The case of double foot-point excitation is most interesting, because of the large difference between the short and large distance behavior. The spatial modulation is largest at short distances, but steady, while on the contrary it is localized at the apex of the loops at long distances, but is timedependent. At short distances the short loops show a strongly oscillating component (the magnetic wave pressure), while the density modulation is almost perfectly steady (panel (c)). On the contrary, at large distances (panel (d)) the wave pressure vanishes, but the density modulation oscillates, the waves being generated by the two outward radial flows converging there, which have been generated by the Alfvén wave pressure at shorter distances (Fig. 5). Oscillations of $\mathrm{Ly}_{\alpha}$ intensity have been found in the distance range between 1.5 and $2.2 R_{\mathrm{s}}$ in open regions as well as within coronal streamers (Morgan et al. 2004). They are sporadic both in space (which is compatible with an occurrence limited to the apex of long loops), and time, which, if generated by Alfvén waves, would imply that a correlated excitation at corresponding foot-points is sporadic too, which would not be unreasonable. When the wave frequency is modulated as in run $\mathrm{U} 3$, then the density in the excited (short) loop reflects the modulation of the forcing, that is, the spatial phase of the density pattern reflects the back and forth oscillations of the magnetic pattern along the loop (panel (e)).

In conclusion, we have investigated how the corona responds to Alfvén wave injection at the coronal base, in the framework of transparent boundary conditions. A strong response, in the form of ever-growing siphon flow, occurs when both foot-points are excited with equal frequencies and amplitude, and a constant phase difference between the two signals. We have failed to find a quantitative criterion for the phase difference to trigger a siphon flow; a qualitative criterion is that the envelope of the wave pressure pattern along the excited loop ensemble should be strongly asymmetric with respect to the apex of the loop (the equator). The case of waves with time-varying frequency shows that a fast siphon growth does 

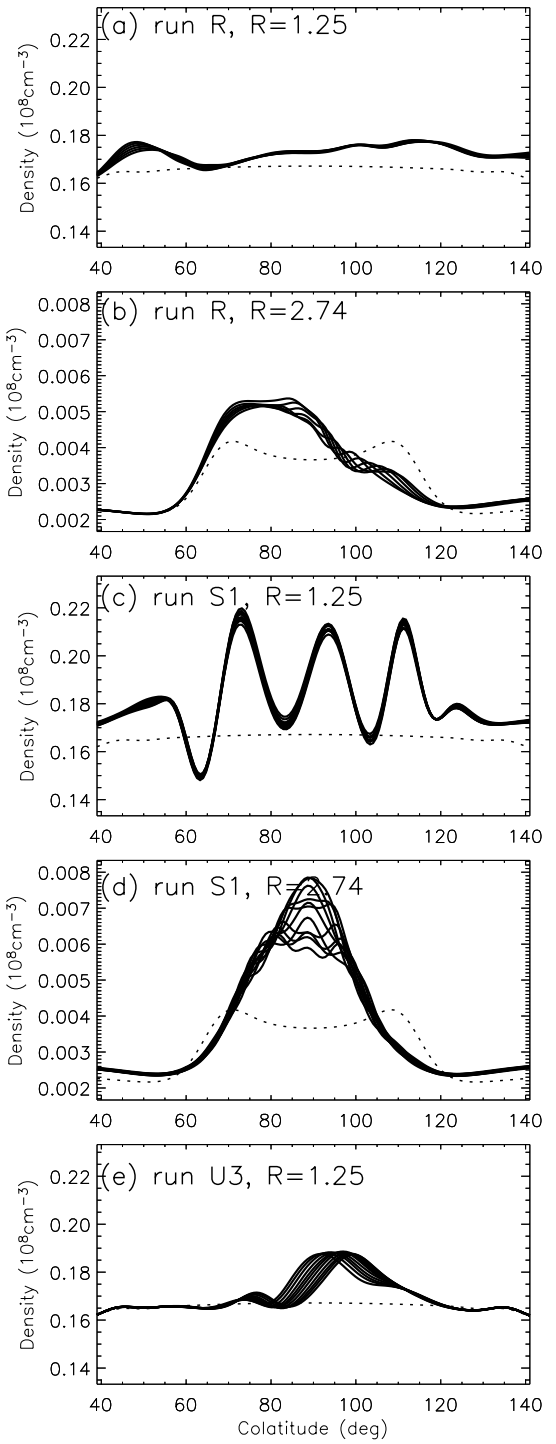

Fig. 14. Density vs. colatitude for several runs and at two heliocentric distances: $r=1.25 R_{\mathrm{S}}$ (apex of loop A), and $r=2.74 R_{\mathrm{s}}$ (apex of loop C). Dotted line: initial profile at $t=0$. Continuous lines: sample profiles during one wave period at $t=0.5$.

not require the phase to be constant: only, the optimal phase difference needs to be realized often enough, which may occur quite generally in a real configuration with random excitation.

The real importance of Alfvén wave excitation in triggering observable modifications of the corona depends on several other factors which remain to be investigated. First, admittedly, the Alfvén wave amplitude likely varies from one foot-point to the other on the Sun. Hence the evolution in general could be intermediate between the case of single injection (slowdown of acceleration in long loops) and double injection (continual acceleration in short loops). Second, and most importantly, the systematic growth of the siphon flow would probably saturate in real solar conditions, due to the dense photosphere, which is not taken into account in the present simulations, due to our choice of transparent boundaries. The best way to solve this issue would be to include the chromospheric transition in the simulation domain, which we postpone for future work. Last, we have studied only very long loops, with heights between typically $0.3 R_{\mathrm{s}}$ and $2 R_{\mathrm{s}}$; an interesting issue is whether smaller loops would still show the same behavior, and how this behavior scales with the wave frequencies. This clearly requires the increase of the numerical resolution in the low atmosphere.

Acknowledgements. One of us (R.G.) thanks Guillaume Aulanier and André Mangeney for helpful discussions. We thank I.D.R.I.S. for providing computer resources (project 040219).

\section{References}

Aulanier, G., Démoulin, P., \& Grappin, R. 2005, A\&A, 430, 1067

Gamet, L., Ducros, F., Nicoud, \& F., Poinsot, T. 1999, Int. J. Numer. Meth. Fluids, 29, 159

Grappin, R., Léorat, J., \& Buttighofer, A. 2000, A\&A, 362, 342

Grappin, R., Léorat, J., \& Habbal, S. 2002, J. Geophys. Res., 107, SSH 16-1, CiteID 1380

Grappin, R., Léorat, J., \& Habbal, S. 2003a, AIP Conf. Proc., 679, 277

Grappin, R., Léorat, J., \& Ofman, L. 2003b, AIP Conf. Proc., 679, 750

Lele, J. 1992, Comput. Phys., 103, 16

Le Saout, J.-M. 2003, Étude numérique d'un modèle de vent solaire, in rapport de stage de DEA, École Centrale de Nantes

Meyer, F., \& Schmidt, H. U. 1968, Z. Angew. Math. Mech., 48, 218

Morgan, H., Rifai Habbal, S., \& Li, X. 2004, ApJ, 605, 521

Patsourakos, S., Klimchuk, J. A., \& MacNeice, P. J. 2004, ApJ, 603, 322

Pneuman, G. W., \& Kopp, R. A. 1971, Sol. Phys. 18, 258

Robb, J. D., \& Cally, P. S. 1992, ApJ, 397, 329

Spadaro, D. 2003, Mem. S. A. It. Suppl., 3, 30

Spadaro, D., Lanza, A. F., Lanzafame, A. C., et al. 2003, ApJ, 382, 486

Velli, M. 1994, ApJ, 432, L55 